\title{
Clippers (Chronic Lymphocytic Inflammation with Pontine Perivascular Enhancement Responsive to Steroids) Management Experience at a single Third-level Medical Center in México. Brenda Bertado Cortes ${ }^{1 *}$, Brian Madariaga Cortes ${ }^{3}$, Massiel Zenteno Zenteno ${ }^{4}$, and Bayron Alexander Sandoval Bonilla ${ }^{2}$
}

${ }^{1}$ Coordinator of Demyelinating Diseases Program. Department of Neurology, National Medical Center XXI Century, Specialty Hospital. Instituto Mexicano del Seguro Social (IMSS).

${ }^{2}$ Coordinator of Brain Tumor and Epilepsy Surgery Program. Department of Neurosurgery. National Medical Center XXI Century, Specialty Hospital. Instituto Mexicano del Seguro Social (IMSS).

${ }^{3}$ Second year resident. Department of Neurology, National Medical Center XXI Century, Specialty Hospital. Instituto Mexicano del Seguro Social (IMSS).

${ }^{4}$ Third year resident. Department of Neurosurgery. National Medical Center XXI Century, Specialty Hospital. Instituto Mexicano del Seguro Social (IMSS).

*Corresponding Author: Brenda Bertado Cortes, Coordinator of Demyelinating Diseases Program. Department of Neurology, National Medical Center XXI Century, Specialty Hospital. Instituto Mexicano del Seguro Social (IMSS).

Received date: January 07, 2021; Accepted date: January 29, 2021; Published date: February 05, 2021

Citation: Brenda B. Cortes., Brian M. Cortes., Massiel Z. Zenteno., and Sandoval Bonilla BR. (2021) Clippers (Chronic Lymphocytic Inflammation with Pontine Perivascular Enhancement Responsive to Steroids) Management Experience at a single Third-level Medical Center in México. J. Neuroscience and Neurological Surgery. 8(2); DOI:10.31579/2578-8868/154

Copyright: () 2021 Brenda Bertado Cortes, This is an open-access article distributed under the terms of The Creative Commons Attribution License, which permits unrestricted use, distribution, and reproduction in any medium, provided the original author and source are credited

\begin{abstract}
:
Chronyc lymphosytic inflammation with pontine perivascular enhacement responsive to steroids (CLIPPERS), described for the first time in 2010 by Pittock and collaborators, is a rare inflammatory disease of the central nervous system (SNC) characterized by the presence of punctuate or curvilinear lesions described like "salt and pepper" appearance on the magnetic resonance imaging (MRI), that enhance with Gadoliniumn administration; this lesions show an exquisite response to corticosteroid therapy.

The etiology of this disease remains unknown. However, the existing articles suggest an autoimmune component, which may or may not be related to other autoinmmune, infectious or malignant pathologies.

Due to the above, it is generally considered that in order to reach the diagnosis of CLIPPERS, the possibility of other more common or aggressive pathologies must first be ruled out through extensive investigation. However, if the clinical and imaging presentation are typical and there is a very high suspicion of CLIPPERS, early initiation of glucosteroid therapy may be recommended. There is no unanimous therapy plan due to the few cases reported worldwide thus far. In cases of severe relapses or atypical behavior, both clinical and radiological, it is recommended to consider a stereotactic biopsy of the lesion to integrate the definitive diagnosis.

Because it is a rare disease, it is relatively unknown to first- contact medical personnel; in our particular case, this leads to referral to the neurosurgery department who, thanks to their experience, have considered a demilinizing disease as a diagnostic possibility and requested an assessment by the neurology department.

Here, we report the evolution and management of three diagnosed cases of CLIPPERS at a single Third-level Medical Center in México; based on clinical, radiological and neuropathological findings; as well as highlighting the importance of lesion biopsy in selected cases.

Keywords: clippers; clinical evolution and management; primary tumors of the central nervous system; biopsy surgery for brainstem lesions; image-guided stereotactic biopsy.
\end{abstract}

\section{Introduction}

CLIPPERS is a rare chronic inflammatory neurological syndrome that affects multiple regions of the brain, including the brainstem, cerebellum, and spinal cord; which has gained recognition since its introduction in 2010 by Pittock [1]. The distinctive finding of CLIPPERS is the presence of multiple punctate curvilinear lesions that show a markedly enhancement "in salt and pepper" pattern, located at the pons and other parts of the brainstem on a magnetic resonance imaging (MRI) [2]. A contrast-like enhancement of the dotted type has also been described in the nuclei of the base, the corpus callosum and the spinal cord. There is a positive clinical and radiological response to corticosteroids treatment [ 3 ]. Differential diagnosis include neurosarcoidosis, primary malignant neoplasms of the central nervous system, vasculitis, lymphoma, and encephalitis [6]. 
CLIPPERS generally appears between the age of 13 and 78 years with no predilection for sex. Common presenting symptoms include progressive gait ataxia and dysarthria [1,4]. Other symptoms include facial paresthesia, diplopia and myelopathy [5].

Histopathologically, it is characterized by a distinctly predominant CD3/CD4 positive T cell lymphocytic perivascular infiltration with white and gray matter compromise, as well as meningeal inflammation with no involvement of the vascular wall. [5-6].

For the definitive diagnosis of CLIPPERS, Tobbin and collaborators have proposed diagnostic criteria consisting on the following.

Clinical characteristics: Subacute cerebellar dysfunction, which responds initially to the use of steroids, without involvement of peripheral nervous system, and without the presence of another pathology that explains the symptoms [6].

Radiological characteristics: homogeneous nodular gadoliniumenhancement, absence of ring enhancement, lacking mass effect predominantly in the pons and cerebellum, smaller than $3 \mathrm{~mm}$ in diameter, decrease or disappearance with the use of steroids, presence of punctate or curvilinear hyperintense lesions on T2 and FLAIR [5-6].

Neuropathological characteristics: Predominant perivascular lymphocytic inflammation and diffuse parenchymal infiltration mostly in white matter and possible gray matter involvement, without myelin loss, dominance of CD 3-positive lymphocytic infiltrate, CD4 and CD8 may also be present, as well as macrophages. The bilateral location of the disease is an important feature that points to CLIPPERS as a diagnosis. Patients with unilateral lesions should be carefully evaluated for alternative causes, such as neoplasms or granulomatous diseases [6].

As previously mentioned, corticosteroid treatment generates a significant reduction in pontine lesions size. A five-day course of daily intravenous methylprednisolone is recommended, followed by a prolonged treatment of $1 \mathrm{mg} / \mathrm{kg}$ oral prednisone daily, with adequate precautions regarding the prevention of side effects. Steroid-sparing agents, such as methotrexate or azathioprine, can be started at the same time as oral prednisone and generally require higher doses [1]. It is known that the disease has a relapsing/remitting course, for which methotrexate is added weekly to treat the relapse event, treatment with anti-CD 20 agents (Rituximab) has now been incorporated, achieving an adequate clinical and radiological response [7, 9]. Oral steroid treatment leads to complete resolution of the improved lesions, producing gradual atrophy of the affected regions and resolution of clinical symptoms [10-11].

We present our experience of three patients who were initially evaluated by the Brain Tumor Program of the Neurosurgery Department in our hospital, due to initial suspicion of central nervous system neoplasia. Subsequently, CLIPPERS diagnosis was concluded by clinical evolution, radiological and neuropathological study. We describe the evolution and management of these cases by the Demyelinating Diseases Program of the Neurology Department in our Hospital. Clinical, radiological and histopathological data were collected from clinical records.

\section{Case 1}

A 44-year-old female patient who started on January 22, 2014; with a clinical presentation characterized by left pulsatile hemicranial headache, accompanied by bilateral incomplete CN III paresis and pancerebellar syndrome, for which she was evaluated and referred to the Neurosurgery Department in a Second-level Hospital, with a possible diagnosis of a pontine region tumor lesion. The magnetic resonance imaging study with gadolinium showed punctuate lesions at the bilateral pontine level, hypointense in T1, hyperintense in T2 and punctiform in FLAIR, with gadolinium enhancement, seen in (figure 2).

Due to high suspicion of demyelinating disease, evaluation of the case is requested to the Neurology Department. It was decided to start treatment with methylprednisolone $1 \mathrm{~g}$ for 5 days and continuing maintenance with oral prednisone after the acute treatment with methylprednisolone. The patient showed clinical improvement and it was observed a significant decrease in brain stem lesions in the follow up MRI. One year later, she developed a relapse with the same symptoms and a stereotactic biopsy of the relapsing lesions was requested at our Center. Histopathological sample showed the presence of an inflammatory process with perivascular lymphocytic infiltrate of predominant T CD3 lymphocytes, supporting the diagnosis of CLIPPERS. Mycophenolic acid $1 \mathrm{~g}$ every 12 hours is added to the treatment and currently, no clinical relapse or new radiological lesions have been documented, this can be seen in (Figure 1), where the evolution and the number of relapses it has presented is summarized.

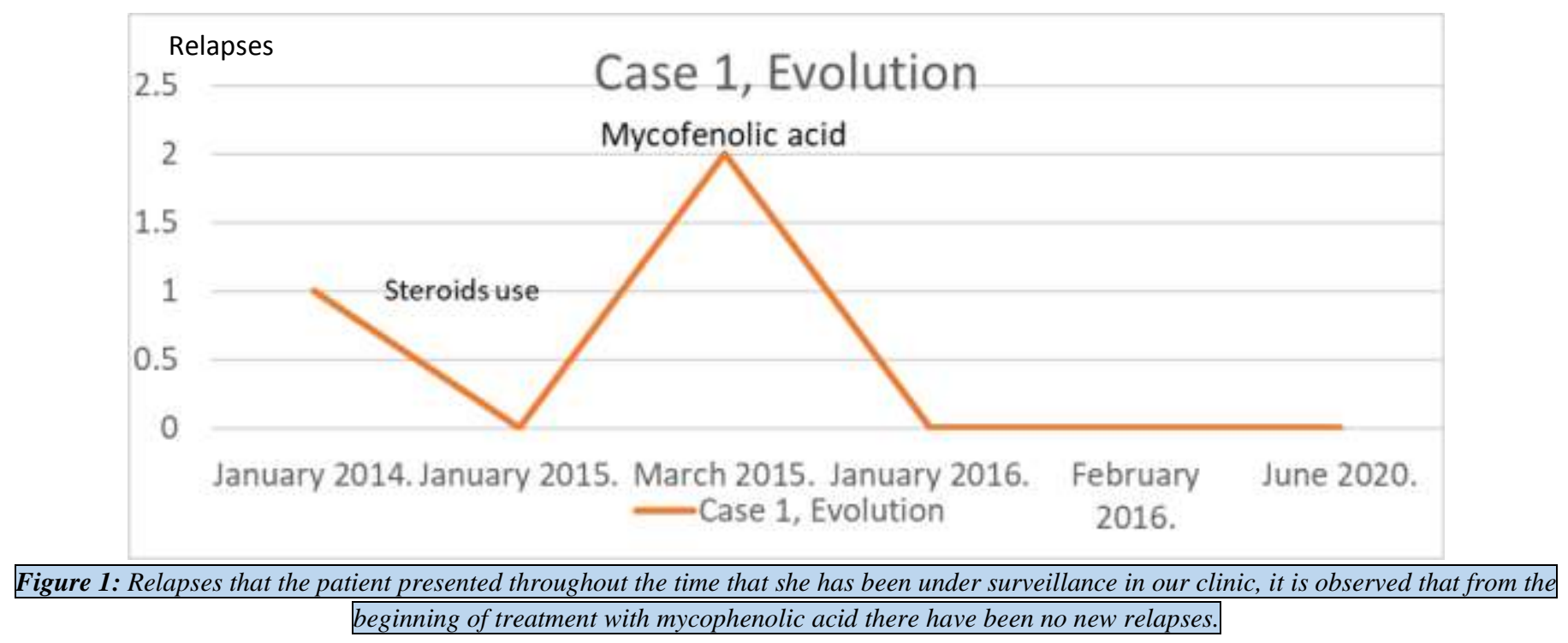




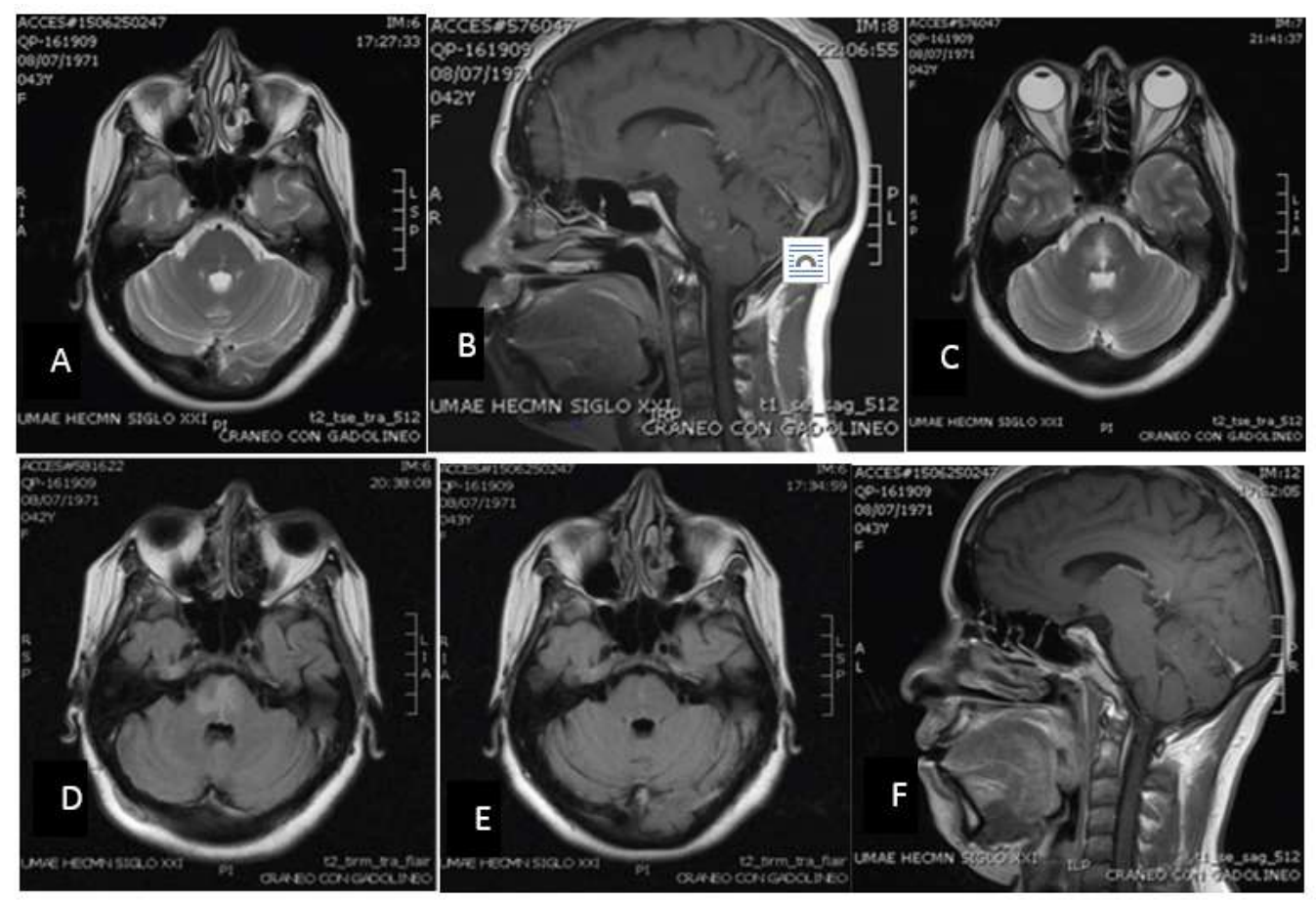

Figure 2: MRI of the brain in axial and sagittal slices of case 1, in images A-C we can see the lesions of the pons with perivascular enhancement. The $D$-F images show the subsequent control by Magnetic Resonance of the steroid treatment and the treatment with mycophenolic acid already established, without evidence of lesions in axial and sagittal sections, without the presence of enhancement with the contrast medium.

\section{Case 2}

A 56-year-old female started with neurological symptoms in October 2014 , characterized by the presence of binocular diplopia in horizontal gaze due to $\mathrm{CN}$ VI paresis in addition to the presence of vertigo and gait ataxia. A MRI study with gadolinium of the brain was performed in which lesions at the level of the pons and bilateral midbrain where evident, hypointense in T1 and hyperintense in T2 and FLAIR, homogeneously enhancing with gadolinium. A high-grade glioma of the brainstem was initially considered for differential diagnosis and was evaluated by the Neurosurgery Department of our Center. Demyelinating disease was also suspected, requesting assessment of the case to the Neurology Department. Clinically, the patient showed a subacute course of cerebellopontine dysfunction, with hyperintense lesions at the pontine and mesencephalic level on T2 and FLAIR and with contrast enhancement on
MRI. CLIPPERS diagnosis was suspected and a methylprednisolone $1 \mathrm{~g}$ every 24 hours for 5 days treatment was started, presenting in a matter of weeks clinical improvement with disappearance of lesions in follow up MRI. A year later, in 2015 she presented with clinical relapse characterized by ataxic gait and it was decided to perform a stereotactic biopsy of the pontine lesion with histopathological findings of an inflammatory infiltrate with a predominance of $\mathrm{T}$ lymphocytes, confirming the diagnosis of CLIPPERS. Treatment with Rituximab was started with cycles of $2 \mathrm{~g}$ every 6-month. Afterwards, in 2018, the patient presented a new relapse of symptoms with pancerebellar syndrome, can be seen in Fig4; which clinically improved with the use of methylprednisolone $1 \mathrm{~g} / 5$ doses and remained stable with Rituximab in an already established dose. Currently, she has showed no additional relapse of the disease, as shown in figure 3 . 


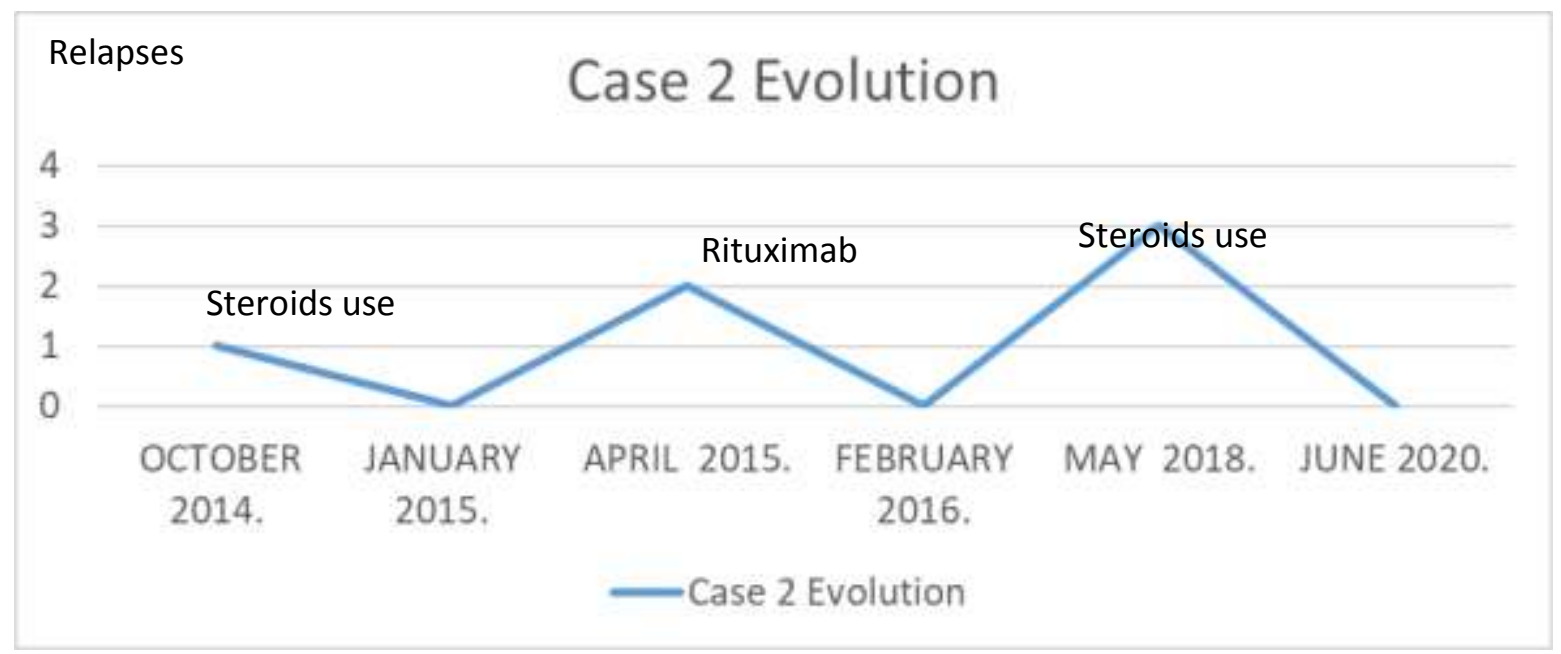

Figure 3: Relapses that patient 2 presented throughout the time that she has been under surveillance in our clinic, it is observed that since the start of treatment with Rituximab, since then only presenting a single relapse.

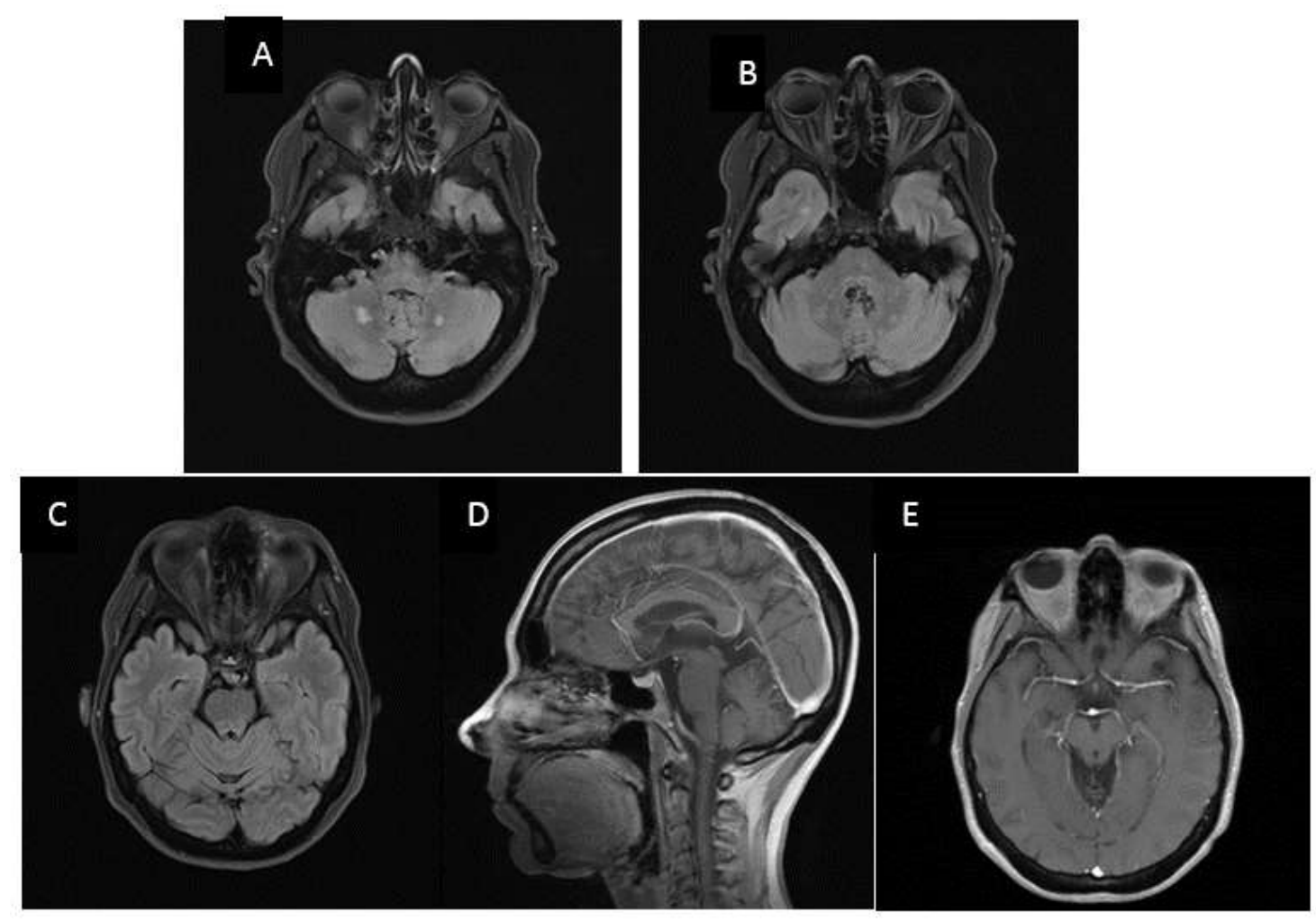

Figure 4: MRI of the brain in axial slices of patient 2, in images $A$ and $B$ we can see the lesions at the bilateral cerebellar level, which were presented in the relapse of 2018.The C-E images show the posterior control of the MRI of the steroid treatment, without evidence of lesions in axial and sagittal slices, without the presence of enhancement with the contrast medium.

\section{Case 3}

A 25-year-old male patient started on November 25, 2019 with a right hemispheric cerebellar syndrome with an insidious instauration during the previous month. An initial diagnostic approach was started with a contrast MRI of the brain where a lesion was identified in the mesencephalon and bilateral pontine tegmental region, isointense in $\mathrm{T} 1$, hyperintense on $\mathrm{T} 2$ and FLAIR, with gadolinium enhancement, observed in the images of Figure 6.

Formerly evaluated by the Brain Tumor Outpatient Appointment Program, hospitalization of the patient was recommended to complete a diagnostic protocol, and treatment with methylprednisolone $1 \mathrm{~g}$ for 5 days is started with clinical improvement and a decrease of about $70 \%$ of previous imaging findings on follow up MRI. Management with oral 
steroids was started and was referred to the Demyelinating Diseases Program of our Center. During follow up, the patient has shown clinical improvement responding to oral steroid dose adjustment. A control MRI was requested with no evidence of previous findings. CLIPPERS diagnosis was integrated by clinic, imaging studies and significant response to steroids. Currently, the patient is under surveillance without new relapse at the moment, as it is graphed in Figure 5. Additional management with immunotherapy will be assessed.

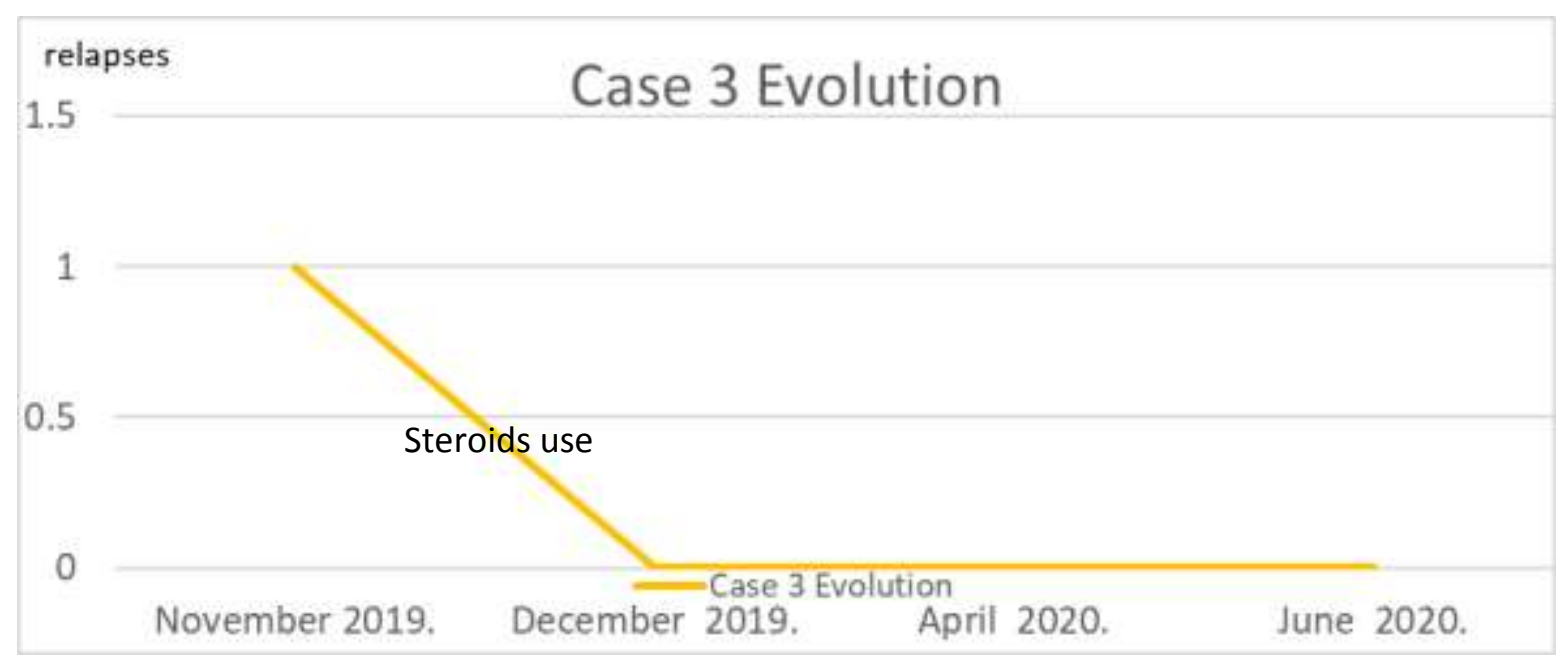

Figure 5: Relapses that patient 3 presented throughout the time that he has been under surveillance in our clinic, it is observed that since the start of treatment with steroids he has no longer presented a single relapse.
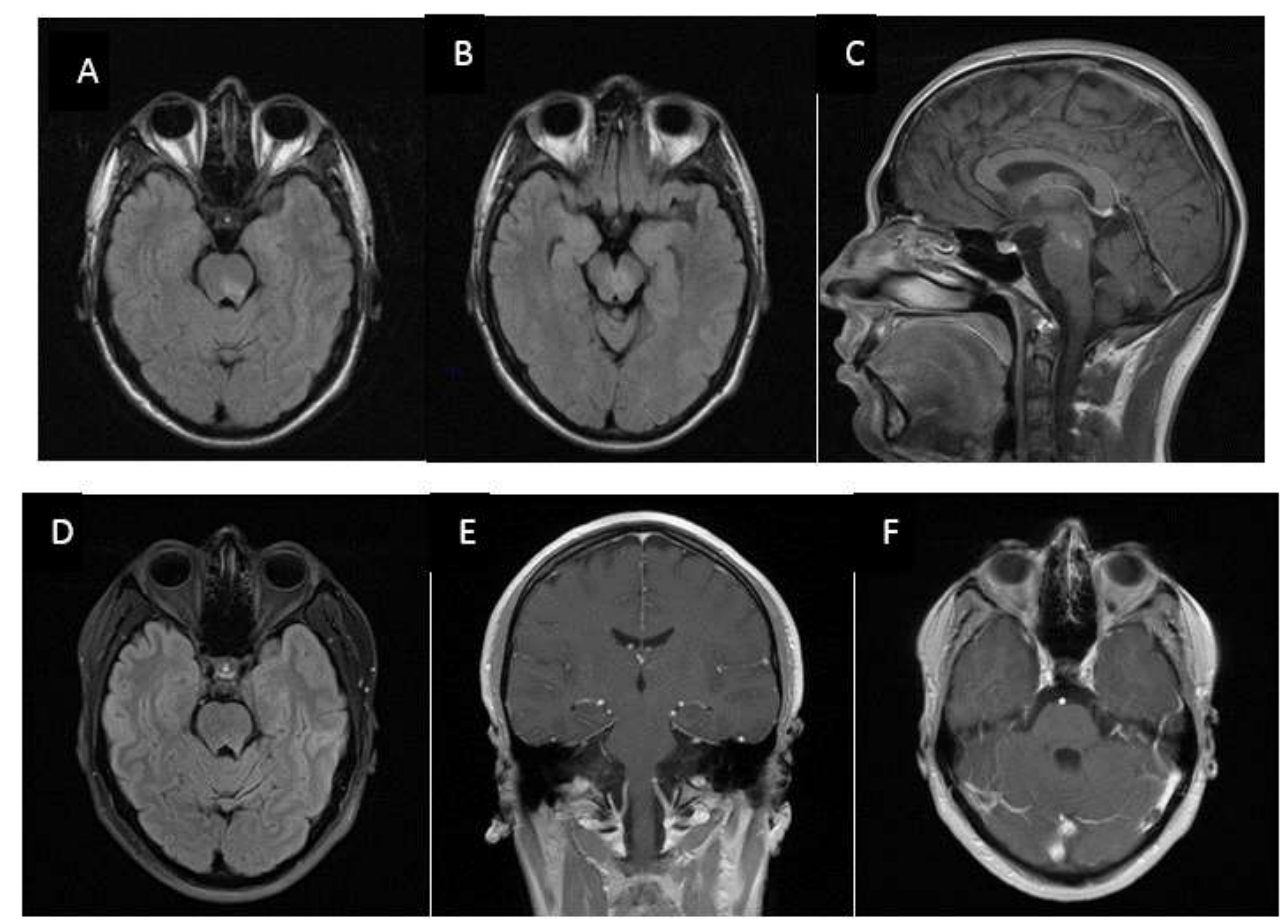

Figure 6: MRI of the brain in axial and sagittal slices of patient 3 in images A-C we can see the lesions at the pontine and mesencephalic level, with enhancement with gadolinium, which appeared at the beginning of his condition. The C-F images show the posterior control of the MRI of the steroid treatment, without evidence of lesions in axial and coronal slices, without the presence of enhancement with the contrast medium.

\section{Discussion}

CLIPPERS is a CNS inflammatory disorder with an uncertain etiology, multiple hypothesis have been proposed however there is not a definitive consensus. Diagnostic uncertainty remains, due to a lack of definitive diagnostic markers. Diagnostic approach must be performed in order to differentiate from primary malignant neoplasms of the central nervous system, demyelinating diseases, autoimmune diseases, among other pathologies. All diagnostic resources should be applied, such as, but not limited to, serological immunological markers, cerebrospinal fluid cytology, as well as an intentional search for infectious pathologies. Tobbin and collaborators proposed diagnostic criteria which include 
clinical, radiological and neuropathologic characteristics, which can serve as a reference to reach the CLIPPERS diagnosis. This condition has symptoms related to the brain stem, cerebellum or both with a very characteristic initial response to steroid use; however, patients require prolonged therapy with steroids and immunosuppressants to avoid relapses and the duration of such treatment remains unknown.

In our Center a total of 3 patients in a period of 6 years arrived with subacute symptoms of cerebellar dysfunction, along with punctiform lesions in the brainstem that show gadolinium enhancement in the magnetic resonance imaging. They were initially assessed by the Brain Tumor Program and a diagnostic protocol was started; the patients presented a favorable response to steroids, we administered methylprednisolone $1 \mathrm{~g}$ for 5 days and continuing maintenance with oral prednisone. Patients were subsequently referred to the Demyelinating Diseases Program and a CLIPPERS diagnosis was integrated in the first 2 cases, upholding the diagnosis with neuropathology. In these cases, a noticeable initial response to steroids had been presented, however, relapses appeared during their clinical evolution and the use of immunotherapy was required.

In first case, the patient achieved clinical and radiological inactivity after the establishment of immunotherapy. In the second case, after immunotherapy treatment only one relapse was present -predominantly cerebellar- which yielded with acute steroid treatment and subsequent maintenance of immunotherapy. Currently, the patient is free of relapses for 2 years. Our last patient presented with a 1-year clinical picture with an excellent response to steroids treatment. A close follow-up was maintained and an early start of immunotherapy has been considered.

Currently in our center, chronic glucocorticosteroid therapy and immunosuppressive agents are used as the standard treatment in this pathology, with adequate response so far. There are not enough cases and studies to determine the duration or to recommend a specific immunosuppressive treatment.

\section{Biopsy rationale.}

Biopsy surgery for brainstem lesions was historically associated with an unacceptably high mortality and morbidity. In general, the vast majority of brainstem lesions are not amenable to surgical resection, especially in case of diffuse lesions, where the difficulty in identifying and planning a reliable preoperative target may become an issue.

Image-guided stereotactic biopsy for histopathological diagnosis of cerebral lesions has become a standard component of the neurosurgical armamentarium; particularly in adults where the diversity of pathology is broader.

Regarding rates for transient and permanent neurological deficits in lesions in the pons, were only slightly higher than with superficial lesions. A recent meta-analysis demonstrated that histological diagnosis could be obtained in $96.2 \%$ of the biopsies. Overall and permanent morbidity were $7.8 \%$, with a mortality rate of $0.9 \%$.

To date, as previously mentioned, validated diagnostic criteria for CLIPPERS are not available, due to the varied clinical presentation and the potential for diagnostic confusion. Until now, 23 atypical CLIPPERS presentations had been described that have contributed to expanding the clinical spectrum of this pathology.
Simon et al. has highlighted core features of CLIPPERS including clinical, radiological, therapeutic response and histopathological criteria. In "typical cases", Pittock et al. suggested a non-invasive approach, who started treatment in $50 \%$ of patients without biopsy.

There is an issue about the appropriate time to take the biopsy. Reluctance can be legitimate considering the potential risks of the procedure and the lack of specificity of histopathologic findings in CLIPPERS; however, it could be the only way to save an early diagnosis of such an underlying serious disease.

In literature, there are descriptions of some conditions which clinically and radiologically seemed to be compatible with CLIPPERS but were only detected by biopsy [e.g. primary CNS lymphoma, lymphomatoid granulomatosis, low-grade glioma and primary angiitis of CNS]. Performance of a brainstem biopsy should therefore be recommended in cases which exhibit one or more of the following attributes: if alternative etiologies remain a distinct possibility despite rigorous investigations, uncommon or atypical clinical/MRI-findings are noticed and resistance to glucosteroid treatment is evident. In these cases, brainstem biopsy may finally add the definitive support. In some cases, CLIPPERS was the preceding diagnosis several months before a B-cell lymphoma.

Therefore, it is recommended to intentionally explore some neuroimaging hints suggestive of an alternative diagnosis which may appear during a latter course of the disease:

- Persistence or recurrence of initial enhancing lesions, with or without increased number of punctate and curvilinear gadolinium-enhanced hyperintense lesions.

- Initial enhancing lesions becoming confluent/lager (size over $3 \mathrm{~mm})$.

- Mass effect with an non-homogeneous or homogenous contrast-enhancement pattern.

- Lesions having characteristics of asymmetry

- $\quad$ Ring like lesion with peripheral gadolinium enhancement and a non-enhanced center.

- Similar imaging characteristics in post gadolinium T1 and T2.

- MRS spectroscopy showing elevated Cho/NAA ratio, as well as high in-phase resonance of lipids and lactates.

\section{Conclusions}

In our experience, it has been extremely useful for the Brain Tumor Program recognizing CLIPPERS as a differential diagnosis of primary neoplasms of the Central Nervous System, especially if located in brainstem and cerebellar regions. Identifying such type of patients allows us to carry out an adequate clinical approach and implement treatment quickly. At the moment there is no well-established therapeutic plan, however, treatment based on steroids and immunosuppressors is scientifically accepted.

The performance of a brainstem biopsy in 2 of our patients has been fundamental for an adequate differentiation of this pathological identity. Especially considering that aggressive pathologies such as Lymphoma can mimic the clinical manifestations of CLIPPERS, as well as it response with glucocorticoid therapy, the relation between this two diseases remains unclear, but several cases have been reported in the literature. In our center, a biopsy was taken early in the course of the disease due to the relapse of the disease in spite of glucocorticoid treatment and risk age group of our patients for other pathologies. 
We consider that this is where the importance of follow-up with long-term serial magnetic resonance studies of patients with a diagnosis of CLIPPERS lies.

Stereotactic brainstem biopsy of unclear inoperable lesions in deep and midline structures can be performed in experienced centers, irrespective of the patient's age or preoperative Karnofsky performance status scale index, with low morbidity and mortality. Even though neuropathological findings are essential for the diagnosis of definite CLIPPERS, we agree that biopsy should be limited to those cases with atypical behavior where an alternative pathology is strongly suspected and possible differential diagnosis must be excluded.

\section{Bibliography}

1. Chronic lymphocytic inflammation with pontine perivascular enhancement responsive to steroids (CLIPPERS) Pittock SJ, Debruyne J, Krecke KN, et al. Brain. 2010;133:2626-2634.

2. Cutting-edge questions about CLIPPERS (chronic lymphocytic inflammation with pontine perivascular enhancement responsive to steroids) Keegan BM, Pittock SJ. Arch Neurol. 2012;69:819-820.

3. Three cases of CLIPPERS: a serial clinical, laboratory and MRI follow-up study. Kastrup O, van de Nes J, Gasser T, Keyvani K. J Neurol. 2011;258:2140-2146.

4. Long-term outcomes of CLIPPERS (chronic lymphocytic inflammation with pontine perivascular enhancement responsive to steroids) in a consecutive series of 12 patients. Taieb G, Duflos C, Renard D, et al. Arch Neurol. 2012;69:847855.

5. Expanding the clinical, radiological and neuropathological phenotype of chronic lymphocytic inflammation with pontine perivascular enhancement responsive to steroids (CLIPPERS) Simon NG, Parratt JD, Barnett MH, et al. J Neurol Neurosurg Psychiatry. 2012;83:15-22.

6. Diagnostic criteria for chronic lymphocytic inflammation with pontine perivascular enhancement responsive to steroids. Tobin WO, Guo Y, Krecke KN, et al. Brain. 2017;140:2415-2425.

7. CLIPPERS: induction and maintenance of remission using hydroxychloroquine. Tan BL, Agzarian M, Schultz DW. Neurol Neuroimmunol Neuroinflamm. 2015;2:0.

8. An extended chronic lymphocytic inflammation with pontine perivascular enhancement responsive to steroids phenotype. Lane C, Phadke R, Howard R. BMJ Case Rep. 2014;2014:0.
9. Effective antituberculous therapy in a patient with CLIPPERS: new insights into CLIPPERS pathogenesis. Mélé N, Guiraud V, Labauge P, Oppenheim C, Mas JL, Taieb G. Neurol Neuroimmunol Neuroinflamm. 2014;1:0.

10. Response to immunotherapy in CLIPPERS: clinical, MRI, and MRS follow-up. Sempere AP, Mola S, Martin-Medina P, Bernabeu A, Khabbaz E, Lopez-Celada S. J Neuroimaging. 2013;23:254-255.

11. Radiologically compatible CLIPPERS' may conceal a number of Pathologies, Jones JL, Dean AF, Antoun N, Scoffings DJ, Burnet NG, Coles AJ. Brain 2011; 134: e187.

12. Stereotactic biopsy of brainstem lesions: Techniques, efficacy, safety, and disease variation between adults and children: A single institutional series and review. Journal of neurosciences in rural practice, Manoj, N., Arivazhagan, A., Bhat, D. I., Arvinda, H. R., Mahadevan, A., Santosh, V., Devi, B. I., Sampath, S., \& Chandramouli, B. A. (2014), 5(1), 32-39.

13. Frame-based stereotactic biopsy of deep-seated and midline structures in 511 procedures: feasibility, risk profile, and diagnostic yield. Hamisch, C.A., Minartz, J., Blau, T. et al. Acta Neurochirurgica 161, 2065-2071 (2019).

14. CLIPPERS: chronic lymphocytic inflammation with pontine perivascular enhancement responsive to steroids. Review of an increasingly recognized entity within the spectrum of inflammatory central nervous system disorders. Clinical and experimental immunologyDudesek, A., Rimmele, F., Tesar, S., Kolbaske, S., Rommer, P. S., Benecke, R., \& Zettl, U. K. (2014), 175(3), 385-396.

15. CLIPPERS: A case report with radiology, three serial biopsies and a literature review. Clinical Neuropathology. Turnquist C, Pretorius PM, DeLuca GC, et al. 2020 Jan/Feb;39(1):19-24.

16. Chronic lymphocytic inflammation with pontine perivascular enhancement responsive to steroids (CLIPPERS) associated with or without lymphoma: Comparison of clinical features and risk factors suggestive of underlying lymphomas, Lu Zhang, Xiao-hang Liu, Fan Jin, Mei-xi Liu, Meng Zhang, Yan Zhang, Dao-bin Zhou, Wei Zhang, Journal of Clinical Neuroscience, Volume 66, 2019, Pages 156-164, ISSN 0967-5868.

17. Unusual CLIPPERS presentation and role of MRI examination in the proper diagnostic assessment: A case report Elena Volaa, Camilla Russoa, Antonio Maceraa, Walter Del Vecchiob, Gianfranco Puotic , Ferdinando Carancid , Andrea Elefantea, European Journal of Radiology Open 6 (2019) 212-214.
This work is licensed under Creative Commons Attribution 4.0 License

To Submit Your Article Click Here: Submit Manuscript

DOI: $10.31579 / 2578-8868 / 153$
Ready to submit your research? Choose Auctores and benefit from:

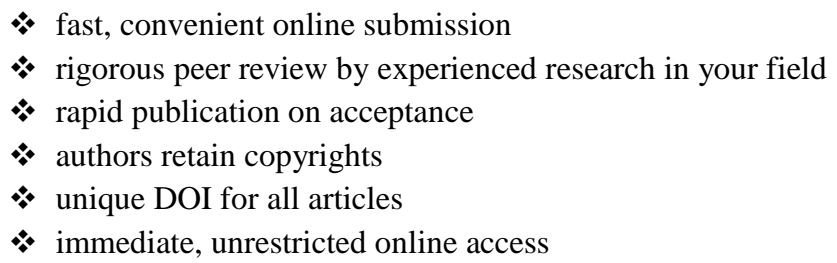

At Auctores, research is always in progress.

Learn more www.auctoresonline.org/journals/neuroscience-andneurological-surgery 